\title{
What new can we learn from cardiac sympathetic neuroimaging in synucleinopathies?
}

\author{
Guillaume Lamotte $^{1,2} \cdot$ David S. Goldstein ${ }^{2}$ \\ Received: 11 February 2022 / Accepted: 12 February 2022 / Published online: 24 February 2022 \\ (c) The Author(s), under exclusive licence to Springer-Verlag GmbH Germany 2022
}

Keywords MIBG $\cdot$ SPECT $\cdot$ PET $\cdot$ Fluorodopamine $\cdot$ Parkinson disease $\cdot$ Multiple system atrophy

\section{Introduction}

Neuroimaging evidence indicating cardiac sympathetic denervation in the Lewy body (LB) synucleinopathy Parkinson disease (PD) and generally intact innervation in the non-LB synucleinopathy multiple system atrophy (MSA) was first reported using ${ }^{18} \mathrm{~F}$-dopamine $\left({ }^{18} \mathrm{~F}\right.$-DA) positron emission tomography (PET) imaging [1]. Numerous studies since then, mainly using ${ }^{123} \mathrm{I}$-metaiodobenzylguanidine ( ${ }^{123}$ I-MIBG) single-photon emission computed tomographic (SPECT) scanning, have confirmed these findings. Of 21 studies in English on this topic involving at least 50 patients, all 21 have reported a significant group difference in the direction of cardiac noradrenergic deficiency in PD (Table 1).

The issue of whether cardiac sympathetic neuroimaging can aid the differential diagnosis of PD vs. MSA seems settled, to the extent that it can be settled. ${ }^{123}$ I-MIBG SPECT scanning for this purpose has been approved for several years in Europe and Asia. In the United States, however, ${ }^{123}$ I-MIBG SPECT scanning is rarely done for this indication, due to lack of reimbursement by third-party payers. Cardiac PET neuroimaging is conduced only in research settings.

Guillaume Lamotte

guillaume.lamotte@hsc.utah.edu

1 Sleep and Movement Disorders Division, Department of Neurology, Movement Disorders and Autonomic Disorders Clinic, University of Utah, Salt Lake City, UT 84108, USA

2 Autonomic Medicine Section, Clinical Neurosciences Program, Division of Intramural Research, National Institute of Neurological Disorders and Stroke, Bethesda, MD 20892, USA

\section{Intra-cardiac regional differences in sympathetic abnormalities}

In this issue of Clinical Autonomic Research, Eckhardt and colleagues report a small, cross-sectional study of patients diagnosed clinically with idiopathic PD or the parkinsonian form of MSA (MSA-P) [2]. Anatomic co-registration of ${ }^{123}$ I-MIBG SPECT images and low-dose chest computed tomography (CT) enabled the examination of regional ${ }^{123}$ I-MIBG-derived radioactivity within the left-ventricular myocardium. Digital thresholding was applied based on voxel intensity to identify 3 different patterns of radioactivity, homogeneous, non-homogeneously reduced, and absent. Among 10 patients with homogeneous radioactivity, 8 had MSA-P and 2 PD; among 7 with non-homogeneously reduced radioactivity, 3 had MSA-P and 4 PD; and among 9 with absent radioactivity, 1 had MSA-P and 8 PD. Nonhomogeneously reduced tracer uptake was most common in the apex and the infero-lateral left-ventricular myocardium.

The same non-homogeneous pattern has been found by ${ }^{18} \mathrm{~F}$-DA [3] and ${ }^{11} \mathrm{C}$-hydroxyephedrine [4] PET scanning in subgroups of $\mathrm{PD}$ patients. The present report confirms these findings using ${ }^{123}$ I-MIBG SPECT.

\section{Relevance of orthostatic hypotension}

In synucleinopathies, a cardinal manifestation of autonomic failure is neurogenic orthostatic hypotension $(\mathrm{OH})$. Abnormal cardiac sympathetic neuroimaging is more consistently found in PD + OH than in PD without $\mathrm{OH}$, by both ${ }^{123}$ I-MIBG SPECT scanning [5] and ${ }^{18} \mathrm{~F}-\mathrm{DA}$ PET scanning [6]. In the study by Eckhardt et al., OH was present in only 9/28 (32\%) patients (5 PD, 4 MSA-P) [2]. This limits the implication of the study for clinical autonomic research, because in autonomic centers, $\mathrm{OH}$ 
Table 1 Studies using cardiac sympathetic neuroimaging in patients with Parkinson disease (PD) and multiple system atrophy (MSA)

\begin{tabular}{llllllll}
\hline PMID & First author & Year & Country & PD N & MSA N & TotalN & ${\text { PD } \neq \text { MSA }^{\text {a }}}^{2}$ \\
\hline 9366173 & Yoshita & 1997 & Japan & 25 & 25 & 50 & Yes \\
10406987 & Orimo & 1999 & Japan & 46 & 7 & 53 & Yes \\
10979878 & Goldstein & 2000 & USA & 29 & 24 & 53 & Yes \\
10853813 & Taki & 2000 & Japan & 41 & 9 & 50 & Yes \\
10872011 & Takatsu & 2000 & Japan & 40 & 12 & 52 & Yes \\
15654042 & Nagayama & 2005 & Japan & 122 & 14 & 136 & Yes \\
20396485 & Shin & 2006 & Korea & 40 & 23 & 63 & Yes \\
16818943 & Kashihara & 2006 & Japan & 130 & 11 & 141 & Yes \\
19418293 & Escamilla-Sevilla & 2009 & Spain & 51 & 9 & 60 & Yes \\
20127386 & Yamashita & 2010 & Japan & 38 & 20 & 58 & Yes \\
21840242 & Kikuchi & 2011 & Japan & 42 & 42 & 84 & Yes \\
22082889 & Kurata & 2011 & Japan & 113 & 16 & 139 & Yes \\
21342781 & Tateno & 2011 & Japan & 90 & 14 & 104 & Yes \\
23613784 & Umemura & 2013 & Japan & 118 & 20 & 138 & Yes \\
26303385 & Mochizuki & 2015 & Japan & 191 & 42 & 233 & Yes \\
27812167 & Fujita & 2016 & Japan & 101 & 21 & 122 & Yes \\
30324423 & Gabilondo & 2018 & Spain & 85 & 40 & 125 & Yes \\
31715596 & Ikeda & 2019 & Japan & 77 & 35 & 112 & Yes \\
34316615 & Sakuramoto & 2019 & Japan & 70 & 16 & 86 & Yes \\
33392714 & Iwabuchi & 2021 & Japan & 90 & 9 & 99 & Yes \\
33981791 & Lenka & 2021 & USA & 50 & 68 & 118 & Yes \\
\hline astan & & & & & & \\
\hline
\end{tabular}

${ }^{\text {a }}$ Statistically significant difference between PD and MSA

${ }^{\mathrm{b}}$ Statistically significant difference between PD and MSA as a group. No difference between PD and MSA-P $(N=19)$. Statistically significant difference between PD and MSA-C $(N=23)$ with central neurodegeneration is often the relevant differential diagnostic issue. A recent retrospective analysis of ${ }^{18} \mathrm{~F}$-DA PET imaging separated patients with $\mathrm{PD}+\mathrm{OH}$ from MSA-P with high sensitivity (92\%) and specificity (96\%) [7].

\section{Functional correlates of cardiac noradrenergic deficiency}

Eckhardt et al. also investigated relationships of ${ }^{123} \mathrm{I}-\mathrm{MIBG}$ imaging with physiological indices of autonomic function. An absence of a blood pressure overshoot above baseline after performance of the Valsalva maneuver, a measure of baroreflex-sympathoneural failure, was associated with low myocardial ${ }^{123}$ I-MIBG-derived radioactivity in PD but not in MSA [2]. This association could reflect postganglionic sympathetic noradrenergic impairment but also central baroreflex-sympathoneural failure.

Eckhardt and colleagues noted a lack of association between cardiac sympathetic innervation and autonomic symptom burden or $\mathrm{OH}$; however, the number of patients with $\mathrm{OH}$ may have been too small to conduct meaningful statistical testing.

\section{Clinical significance of cardiac noradrenergic deficiency}

Inconsistent results have been reported in the literature regarding the association between motor symptoms and cardiac noradrenergic deficiency in PD. The extent to which cardiac noradrenergic deficiency contributes to cardiovascular dysfunction in synucleinopathies is still unclear and requires further research.

Cardiac sympathetic neuroimaging may provide a better understanding of the pathophysiology of other non-motor symptoms, such as anosmia, rapid eye movement behavior disorder, fatigue, and cognitive dysfunction, all of which have been associated with evidence of myocardial noradrenergic deficiency [8]. Mechanisms for the associations between these non-motor symptoms and cardiac noradrenergic deficiency in PD remain unknown. 


\section{Progression biomarkers}

There is a push to develop quantifiable biomarkers of disease progression for use in clinical trials for neurological diseases. Longitudinal studies are far less common than cross-sectional studies such as that by Eckhardt et al. Using ${ }^{18} \mathrm{~F}$-DA PET imaging, we have found that ${ }^{18} \mathrm{~F}$-DAderived radioactivity decreases by a median of $4 \%$ per year in PD [9]. Because of individual variability in the rate of decline, a clinical trial of a disease-modifying approach using ${ }^{18} \mathrm{~F}$-DA-derived radioactivity as an outcome measure would require about 70 patients in each of the experimental treatment and standard treatment groups to detect a 50\% decrease in the loss of cardiac noradrenergic innervation over a 5-year-period, which would represent a substantial study. Thus, cardiac neuroimaging may be more fitted as a secondary outcome or as part of a composite outcome, in which multiple endpoints are combined. Importantly, there is evidence that decreased vesicular uptake in cardiac sympathetic nerves is present upon initial evaluation of patients with LB synucleinopathies and may provide a biomarker of catecholaminergic dysfunction early in the disease process [9].

Neuroimaging evidence of cardiac noradrenergic deficiency can precede striatal dopamine deficiency and the onset of motor signs in PD [10]. In the prospective, longitudinal intramural NINDS PDRisk study, in individuals at high risk for developing PD (at least 3 of the following risk factors: genetic susceptibility, olfactory dysfunction, dream enactment, and $\mathrm{OH}$ ), low cardiac ${ }^{18} \mathrm{~F}$-DA-derived radioactivity has been reported to predict a diagnosis of PD by 3 years of follow-up [11]. The findings indicate the potential of cardiac sympathetic neuroimaging for providing a preclinical biomarker of PD; however, the generalizability to the broader population of individuals with fewer risk factors is unknown.

\section{Dysfunction vs. denervation}

PET imaging agents such as ${ }^{18} \mathrm{~F}$-DA, ${ }^{11} \mathrm{C}$-epinephrine, and ${ }^{11} \mathrm{C}$-phenylephrine are substrates for metabolism by monoamine oxidase (MAO). After uptake into the neuronal cytoplasm, these agents have two alternative fates. The main fate is vesicular uptake; oxidative deamination via MAO is a minor fate. By measuring the deaminated metabolite of ${ }^{18} \mathrm{~F}$-DA simultaneously with ${ }^{18} \mathrm{~F}$-DA, one can examine the efficiency of vesicular sequestration. It was by applying this approach that decreased intra-neuronal vesicular uptake was discovered in LB diseases [6]. Confirmation of this finding has come from tracking the radioactivity during the testing session. $\mathrm{PD}+\mathrm{OH}$ is associated with accelerated loss of ${ }^{18} \mathrm{~F}$-DA-derived radioactivity [12]. The implications for treatment of this functional abnormality are substantial. Put simply, you cannot treat dead neurons, whereas neurons that are dysfunctional but extant ("sick-but-not-dead") might be salvaged.

This functional abnormality cannot be quantified by ${ }^{123} \mathrm{I}$-MIBG SPECT or by ${ }^{18} \mathrm{~F}$-hydroxyephedrine PET, since neither imaging agent is a substrate for MAO. On the other hand, accelerated loss ("washout") of ${ }^{123}$ I-MIBG-derived radioactivity might provide a biomarker of increased sympathetically mediated exocytosis. This possibility can be tested by determining effects of sympatholysis on ${ }^{123}$ I-MIBGderived radioactivity.

\section{PAF as a prototype of "body-first" PD}

A model of brain-first versus body-first subtypes of PD has been proposed by Borghammer and colleagues [13]. In the brain-first (top-down) type, $\alpha \mathrm{S}$ pathology initially occurs in the brain, with secondary spreading to the peripheral autonomic nervous system; whereas in the body-first (bottom-up) type, the pathology originates in the enteric or peripheral autonomic nervous system and then spreads to the brain. The Lewy body form of PAF can evolve to PD or dementia with Lewy bodies (DLB) [14]. Measuring CSF levels of catecholamines and analyzing post-mortem data in autonomic synucleinopathy patients and controls, we reported recently that the synucleinopathies have in common evidence of central noradrenergic deficiency but differ in extents of central dopaminergic deficiency-prominent in PD and MSA, less pronounced in PAF [15]. Therefore, PAF may be the prototype for peripheral-to-central disease progression in LB diseases. Only a minority of PAF patients seem to develop symptomatic central synucleinopathy; however, these patients might be identifiable based on biomarkers of central nervous system involvement, such as by ${ }^{18}$ F-DOPA PET scanning [10] or by brainstem neuromelanin magnetic resonance imaging [16].

\section{PET vs. SPECT imaging}

PET scanning offers several advantages over SPECT scanning. These include better spatial resolution, a smaller amount of injected radioactivity, the possibility of measuring radioactivity concentrations in tissues in absolute terms, and, importantly, the analysis of curves relating tissue radioactivity vs. time (time-activity curves), which provides valuable information about not only innervation but also functional abnormalities in residual nerves, such as a vesicular storage defect [6]. The time frames involved with PET and 
SPECT scanning differ. Injected cardiac sympathetic neuroimaging agents exit the bloodstream almost instantly and are taken up by sympathetic nerves extremely rapidly. Although the initial ${ }^{123}$ I-MIBG SPECT scan, at about 15-30 min, is called "uptake," peak ${ }^{18} \mathrm{~F}$-DA-derived radioactivity normally occurs by about 5 min after tracer injection.

Comparing ${ }^{18} \mathrm{~F}$-DA PET with ${ }^{123} \mathrm{I}$-MIBG SPECT scanning in the same patients with autonomic synucleinopathies would provide valuable information, and we would welcome investigators outside the NIH to join in the IND covering ${ }^{18}$ F-DA PET scanning, with a view toward a multi-center collaborative study.

\section{Implications}

Research on cardiac sympathetic neuroimaging in synucleinopathies is not dead. Early involvement of the autonomic nervous system should be the focus of future studies. In patients for whom the pathophysiological process starts in the periphery and propagates to the brain, understanding and modulating the peripheral process might not only help alleviate the associated non-motor symptoms but also prevent the central degeneration that leads to the motor signs and symptoms. Cardiac sympathetic neuroimaging combined with other measurable and robust biomarkers of preclinical disease may serve as a tool to target catecholaminergic neurodegeneration at the earliest stages of LB diseases.

Acknowledgements Dr. Goldstein's research is supported by the Division of Intramural Research, NINDS, NIH.

Funding No funding to declare.

\section{Declarations}

Conflict of interest The authors declare that they have no competing interest.

\section{References}

1. Goldstein DS, Holmes C, Cannon RO 3rd, Eisenhofer G, Kopin IJ (1997) Sympathetic cardioneuropathy in dysautonomias. N Engl J Med 336(10):696-702

2. Eckhardt C, Krismer F, Donnemiller E, Eschlböck S, Fanciulli A, Raccagni C, Bösch S, Mair K, Scherfler C, Djamshidian A, Uprimny C, Metzler B, Seppi K, Poewe W, Kiechl S, Virgolini I, Wenning GK (2022) Cardiac sympathetic innervation in Parkinson's disease versus multiple system atrophy. Clin Auton Res. Accessed 19 Feb 2022 (ahead of print)

3. Goldstein DS, Holmes CS, Dendi R, Bruce SR, Li ST (2002) Orthostatic hypotension from sympathetic denervation in Parkinson's disease. Neurology 58(8):1247-1255
4. Wong KK, Raffel DM, Koeppe RA, Frey KA, Bohnen NI, Gilman S (2012) Pattern of cardiac sympathetic denervation in idiopathic Parkinson disease studied with $11 \mathrm{C}$ hydroxyephedrine PET. Radiology 265(1):240-247. https://doi.org/10.1148/radiol.12112723

5. Kim JS, Park HE, Park IS, Oh YS, Ryu DW, Song IU, Jung YA, Yoo IR, Choi HS, Lee PH, Lee KS (2017) Normal "heart" in Parkinson's disease: is this a distinct clinical phenotype? Eur J Neurol 24(2):349-356. https://doi.org/10.1111/ene.13206

6. Goldstein DS, Holmes C, Kopin IJ, Sharabi Y (2011) Intra-neuronal vesicular uptake of catecholamines is decreased in patients with Lewy body diseases. J Clin Investig 121(8):3320-3330. https://doi.org/10.1172/JCI45803

7. Lenka A, Lamotte G, Goldstein DS (2021) Cardiac (18)F-dopamine PET distinguishes PD with orthostatic hypotension from Parkinsonian MSA. Mov Disord Clin Pract 8(4):582-586. https:// doi.org/10.1002/mdc3.13190

8. Goldstein DS, Sharabi Y (2017) The heart of PD: Lewy body diseases as neurocardiologic disorders. Brain Res. https://doi.org/ 10.1016/j.brainres.2017.09.033

9. Lamotte G, Holmes C, Wu T, Goldstein DS (2019) Long-term trends in myocardial sympathetic innervation and function in synucleinopathies. Parkinsonism Relat Disord 67:27-33. https:// doi.org/10.1016/j.parkreldis.2019.09.014

10. Goldstein DS, Holmes C, Sewell L, Park MY, Sharabi Y (2012) Sympathetic noradrenergic before striatal dopaminergic denervation: relevance to Braak staging of synucleinopathy. Clin Auton Res 22(1):57-61. https://doi.org/10.1007/s10286-011-0136-4

11. Goldstein DS, Holmes C, Lopez GJ, Wu T, Sharabi Y (2018) Cardiac sympathetic denervation predicts PD in at-risk individuals. Parkinsonism Relat Disord 52:90-93. https://doi.org/10.1016/j. parkreldis.2017.10.003

12. Goldstein DS, Holmes C, Sullivan P, Mash DC, Sidransky E, Stefani A, Kopin IJ, Sharabi Y (2015) Deficient vesicular storage: a common theme in catecholaminergic neurodegeneration. Parkinsonism Relat Disord 21(9):1013-1022. https://doi.org/10. 1016/j.parkreldis.2015.07.009

13. Horsager J, Andersen KB, Knudsen K, Skjaerbaek C, Fedorova TD, Okkels N, Schaeffer E, Bonkat SK, Geday J, Otto M, Sommerauer M, Danielsen EH, Bech E, Kraft J, Munk OL, Hansen SD, Pavese N, Goder R, Brooks DJ, Berg D, Borghammer P (2020) Brain-first versus body-first Parkinson's disease: a multimodal imaging case-control study. Brain 143(10):3077-3088. https://doi.org/10.1093/brain/awaa238

14. Kaufmann H, Norcliffe-Kaufmann L, Palma JA, Biaggioni I, Low PA, Singer W, Goldstein DS, Peltier AC, Shibao CA, Gibbons CH, Freeman R, Robertson D, Autonomic Disorders C (2017) Natural history of pure autonomic failure: a United States prospective cohort. Ann Neurol 81(2):287-297. https://doi.org/10.1002/ana. 24877

15. Goldstein DS, Sullivan P, Holmes C, Lamotte G, Lenka A, Sharabi Y (2021) Differential abnormalities of cerebrospinal fluid dopaminergic vs noradrenergic indices in synucleinopathies. J Neurochem. https://doi.org/10.1111/jnc.15371

16. Doppler CEJ, Kinnerup MB, Brune C, Farrher E, Betts M, Fedorova TD, Schaldemose JL, Knudsen K, Ismail R, Seger AD, Hansen AK, Staer K, Fink GR, Brooks DJ, Nahimi A, Borghammer P, Sommerauer M (2021) Regional locus coeruleus degeneration is uncoupled from noradrenergic terminal loss in Parkinson's disease. Brain 144(9):2732-2744. https://doi.org/10.1093/brain/ awab236 